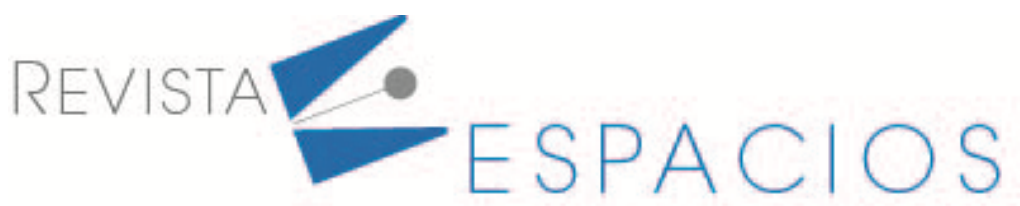

Vol. 42 (20) $2021 \cdot$ Art. 1

\title{
Tipos de comunidades virtuales de aprendizaje en la práctica docente
}

\section{Types of virtual learning communities in teaching practice}

\author{
BOLAÑO García, Matilde ${ }^{1}$ \\ GOYENECHE, León Eduin ${ }^{2}$ \\ DUARTE, Acosta Nixon ${ }^{3}$ \\ VILLALOBOS, Ropain Narlys ${ }^{4}$
}

\section{Resumen}

El presente artículo busca identificar los tipos de comunidades virtuales de aprendizaje existente en la institución educativa No.9, sede Manuel Rosado Iguarán, municipio Maicao, departamento La Guajira, Colombia. Metodológicamente responde a la secuencia teórica del paradigma positivista, para esto se usa la encuesta, se obtuvo como resultado no solo la existencia de los grupos también la preponderancia o afinidad de los docentes. Dando como conclusión una implementación superficial de los CVA.

Palabras clave: comunidad de aprendizaje, práctica docente, enseñanza virtual

\begin{abstract}
This article seeks to identify the types of virtual learning communities existing in educational institution No.9, Manuel Rosado Iguarán headquarters, Maicao municipality, La Guajira department, Colombia. Methodologically it responds to the theoretical sequence of the positivist paradigm, for this the survey is used, as a result not only the existence of the groups was obtained, but also the preponderance or affinity of the teachers. Giving as conclusion a superficial implementation of the CVA.

Key words: learning community, teaching practice, virtual teaching
\end{abstract}

\section{Introducción}

El aprendizaje y el desarrollo de competencias en la labor docente se han convertido en una de las exigencias del actual contexto educativo y del nuevo entorno social del mundo contemporáneo. En este sentido, la construcción

\footnotetext{
1. Doctora en Ciencias de la Educación, Docente e investigadora de educación superior. Universidad del Magdalena. Santa Marta, Colombia. Correo electrónico: mbolano@unimagdalena.edu.co. https://orcid.org/0000-0002-5514-2992

2 Doctor en Ciencias de la Educación, Magíster en Informática Educativa, Licenciado en Educación Básica con énfasis en Informática. Correo electrónico goyeneche31@gmail.com.

3 Magíster en ingeniería en el área de Sistemas y computación, Ingeniero de sistemas y computación, Especialista en construcción de software. Correspondencia: nixon.duarte@uniremington.edu.co. https://orcid.org/0000-0002-2572-3366

4 Especialista, Licenciada en educación básica con énfasis en informática. Universidad del Magdalena, Santa Marta, Colombia. Correo electrónico: nvillalobo@unimagdalena.edu.co
} 
de conocimiento sugiere que la praxis docente asuma novedosas posturas y las transformaciones requeridas como parte del proceso facilitador de su rol profesional.

Al respecto Canales \& Peré (2007), señalan que los docentes deben tener presente que para cualquier tema van a encontrar buenos recursos didácticos, este valor añadido aumenta la funcionalidad de estas plataformas; adicionalmente el autor sostiene, que si bien en estos espacios se ofrecen estos apoyos didácticos, en la red existen variadas páginas web, no específicamente de carácter educativo, que posibilitan la realización de una buena labor formativa.

Según varios estudios descritos por la UNESCO (2013), en América Latina los maestros y profesores han desarrollado las habilidades necesarias para usar estas tecnologías, pero no han transformado estas competencias en prácticas innovadoras en las aulas. Para el caso de Colombia, el Ministerio de Educación Nacional (2012), da inicio a la Estrategia Nacional de Recursos Educativos Digitales Abiertos, con la cual se busca contribuir a mejorar las condiciones de acceso a la información y al conocimiento por parte de las comunidades educativas y fortalecer el uso educativo de las tecnologías de la información y comunicación (TIC). Esto sugiere que la implementación de las TIC en los salones de clases se ha convertido en una variable preponderante cuando se plantean estrategias de innovación e intervención en el campo pedagógico. De tal manera que el ejercicio de la profesión docente sea activo, sujeto a una reflexión permanente para identificar los problemas en el desarrollo de sus prácticas y la búsqueda de soluciones de manera conjunta a través del intercambio de experiencias educativas.

Así pues, la coordinación académica de la Institución Educativa \#9 (I.E.\#9) del municipio de Maicao, departamento La Guajira, Colombia, para el año 2014, evidencia que las prácticas docentes se limitaron al cronograma de actividades propuestos por las directivas, es decir, jornadas pedagógicas periódicas para la evaluación de los procesos de enseñanza y aprendizaje de las diferentes sedes.

Esta panorámica de la realidad educativa de los docentes de I.E. \#9, evidencia el poco aprovechamiento del uso e incorporación de nuevas herramientas, como son las comunidades virtuales de aprendizaje, las cuales, según Cabero, (2006), se conciben como un colectivo de personas, que comparten unos valores e intereses comunes y que se comunican a través de las diferentes herramientas de comunicación que nos ofrecen las redes telemáticas, sean sincrónicas o asincrónicas. Algunas de las características preponderantes en estos mecanismos son que facilitan la comunicación entre los usuarios dejando de lado el factor tiempo y espacio; son lugares en los cuales un grupo de estudiantes o docentes, quienes son sus usuarios, publican e intercambian información; además permite el almacenamiento en múltiples formatos; facilitan el trabajo en grupos pequeños y grandes donde sus usuarios realizan acciones para avanzar en equipo usando diversas herramientas tecnológicas y sus integrantes tienen acceso a una vasta cantidad de recursos compartidos y a parámetros políticos que soportan estos recursos.

Las comunidades virtuales de aprendizaje facilitan la creación de entornos en los cuales el estudiante se sienten a gusto tecnología permite que las prácticas pedagógicas tradicionales pasen a un entorno digital, este escenario es llamativo y brinda un acceso casi ilimitado a la información, sin embargo cuando los docentes desconocen las potencialidades de estos entorno, cortan el desarrollo y el crecimiento, además limitan las posibilidades del aprendizaje, el Ministerio de Educación Nacional (2012) afirma que la ausencia de comunidades virtuales de aprendizaje en el contexto educativo, es un acto de resistencia a la innovación y al desarrollo de las capacidades cognitivas, comunicativas y afectivas.

Cabero (2010), Afirma que las comunidades virtuales de aprendizaje potencian la práctica pedagógica debido a sus carteristas, las cuales son libre acceso y fácil aprensión. Pues esto permite acceder a un escenario en el cual la información está disponible y dada su cualidad colaborativa se construyen los conceptos a partir de la 
experiencia de los integrantes los cuales a su vez responden a las necesidades del estudiante y los objetivos de la comunidad académica. Por otro lado, Tirado \& Martínez (2010), afirman que un valor primordial en las comunidades virtuales es el sentido de la presencia social, el sentido de la cooperación y el valor de la interacción.

Finalmente, el salón de clases se configura como un escenario nuevo, esto debido a la interacción de la praxis pedagógica con la tecnología, esto amplía el abanico de secuencias didácticas del docente ayudando al estudiante con la investigación y resolución de actividades. Cabe destacar que algunos de estos actores desconocían las bondades que las comunidades virtuales de aprendizaje (CVA) ofrecen a la educación, como lo son, acciones formativas enmarcadas en la innovación, la oportunidad de trabajar con mecanismos afines a la juventud como los artefactos tecnológicos, la conformación de grupos de trabajo interdisciplinares, la oportunidad de conocer los distintos contextos que tienen lugar en la vida de los participantes, el seguimiento individual y la creación de una identidad estudiantil digital, en el cual se tomen decisiones entorno a las potencialidades que están a la mano, tanto para estudiantes como docentes, de esta forma se abre el abanico de posibilidades de los actores involucrados en el proceso de enseñanza y aprendizaje.

El ignorar o pasar por alto estas nociones puede afectar la cualificación de los estudiantes ya que desaprovechan, por desconocimiento, la posibilidad de aprender con tecnología de una manera lúdica y atractiva. Por esta razón, se propuso para este estudio identificar los tipos de comunidades virtuales de aprendizaje existentes en la Institución Educativa No.9, sedes Manuel Rosado Iguarán, 20 de Julio, Villa Inés y Gabriel García Márquez del municipio de Maicao, La Guajira, Colombia.

\subsection{Tipos de comunidades virtuales de aprendizaje}

Meirinhos \& Osorio (2009), en su análisis bibliográfico y documental de la taxonomía de las comunidades virtuales de aprendizaje destacan la relevancia de metodologías e instrumentos que soportan de modo eficaz, la facilidad para el desarrollo de actividades de las mismas. Así mismo, los autores basados en los trabajos de DíezPalomar \& García (2010), Dillenbourg y otros (2003), presenta una clasificación de las comunidades virtuales de aprendizaje; la primera referida a la fuerza del vínculo social, mientras la segunda se relaciona con la intensidad de aglutinación de sus participantes.

En este sentido, los miembros que participan en ella emprenden una actividad hacia el cumplimento de metas de aprendizaje, generados a partir del grado de cohesión y vínculo social de sus participantes. Es así como los autores clasifican en cuatro tipos de comunidades virtuales: (a) Comunidades de interés, (b) Comunidades de interés inteligente; (c) Comunidades de aprendices; y (d) comunidades de práctica. A partir de los anteriores planteamientos, se consideró oportuno asumir una postura ecléctica en cuanto a la tipificación de las CVA, pues estos permiten realizar el abordaje de las temáticas de una forma acorde a la realidad del estudiante, a esto se le añade que su componente tecnológico permite la creación de diversos escenarios y grupos de trabajo, cada uno de estos tipos de comunidades virtuales de aprendizaje responde a una necesidad especifica de la institución o colectivo de personas, por consiguiente algunos tendrán mayor relevancia que otros.

\subsection{Comunidades de interés}

Las comunidades de interés se focalizan principalmente en que sus participantes pretenden encontrar un espacio de pertinencia compartido en relación a los gustos y aficiones, sean de naturaleza profesional o lúdica. Otro aspecto relevante es la forma de afiliarse de sus miembros, pues lo realizan de manera voluntaria en virtud de su provecho por compartir información entre unos y otros.

Por su parte Zúñiga, et al., (2010), indican que las comunidades de interés se centran en los objetivos y la forma de difusión del conocimiento, por ello, es un espacio para la discusión de temas en común e interés de 
intercambiar información entre los colaboradores dentro de un contexto de aprendizaje formal o informal en forma rápida; sin embargo, una vez lo han obtenido no utilizan más la comunidad.

\subsection{Comunidades de interés inteligente}

Las comunidades de interés inteligente agrupan personas con una utilidad común en la realización de una tarea específica, reflejada en interacciones que atienden a un trabajo colaborativo, ajustadas a la asignación de roles y funciones en pretensión de la entrega de un producto final.

En esta línea, Gros (2008), agrega que las personas en este tipo de comunidades se asignan o agrupan en función de las tareas sobre un tema, proyecto o problema bien definido con un inicio y un final claro, además se establecen objetivos de aprendizaje como parte del proyecto. Así mismo, se distribuyen los roles y otorgan responsabilidades a sus participantes dentro del grupo. Cabe anotar que a través del proceso de formación del grupo e intercambio de información se crea un conocimiento sistemático.

\subsection{Comunidades de aprendices}

Las comunidades de aprendizaje están remitidas a un grupo de personas con diferentes niveles de experiencia y de conocimiento que aprenden mediante su implicación y participación en actividades relevantes culturalmente, a través de la colaboración que establecen entre sí, a la construcción del conocimiento colectivo que llevan a cabo y a los diversos tipos de ayudas que se prestan mutuamente. Para Gairín (2006), las comunidades de aprendizaje buscan ampliar el intercambio de conocimientos mediante una trama de interacciones entre sus participantes. Por ello, cuando adopta el concepto de aprendizaje a lo largo de la vida y relaciona el aprendizaje no formal e informal, propone nuevos modelos de organización de la educación basados en el compromiso y la corresponsabilidad de los agentes sociales y comunitarios implicados.

Según Coll (2008), la comunidad de aprendices está orientada al desarrollo de un contenido o tarea de aprendizaje específica. Es decir, permite nuevas aproximaciones dinámicas para aprender entre sus participantes a través de la colaboración con otros y aprender de ellos a través de guías o ayudas. Pese a las características de sus miembros que difieren significativamente entre sí y del contexto institucional en el que se encuadran (comunidades de aprendizaje para profesionales, docentes en formación, empleados de una empresa, entre otros).

\subsection{Comunidades de práctica}

Son aquellas formadas por profesionales cuyo objetivo es mejorar el conocimiento sobre su propia práctica. Se fundamentan en la creación de conocimiento a partir de las experiencias de sus miembros, la comunicación eficaz de los métodos y herramientas utilizadas, éxitos, fracasos, entre otros. Este tipo de comunidades cambian y evolucionan constantemente; su éxito está ligado al dinamismo de la actividad en la propia red (Gros, 2008); entre tanto, Meirinhos \& Osorio (2009) consideran que las comunidades de práctica son aquellas cuyo objetivo es mejorar las condiciones de ejercicio de la profesión, a través de la cartilla, del auxilio mutuo y por la construcción de conocimiento, establecidas en procesos de aprendizaje colaborativo.

\section{Metodología}

Esta investigación se desarrolló siguiendo los pasos de una investigación cuantitativa, de tipo descriptiva en consideración a lo planteado por Arias (2012), que permiten medir de forma independiente las variables, aun cuando no se formule hipótesis alguna, para este menester se indica en el párrafo siguiente el objetivo de la intervención. De allí que el tipo de investigación esté referido a escudriñar con cuanta profundidad se abordará el objeto, sujeto o fenómeno a estudiar. 
Es importante indicar que el objetivo de este estudio fue, Identificar los tipos de comunidades virtuales de aprendizaje existente en la institución educativa No.9, sede Manuel Rosado Iguarán, 20 de Julio, Villa Inés y García Márquez del municipio de Maicao la Guajira Colombia. Por ende, los puntos de partida para la recolección, medición y análisis de la información son, las comunidades virtuales de aprendizaje, de igual forma aludiendo a los tipos de grupos que orbitan entorno a esta categoría.

Los datos recolectados a través de una encuesta (Anexo A) fueron procesados mediante la creación de tablas en Excel, cuya organización da lugar a la creación de una serie de cuadros que tienen información de gran relevancia para explicar de forma clara los tipos que se desprenden del proceso metodológico de la intervención

En cuanto a su diseño es no experimental, transeccional descriptivo que, según Hernández, et al. (2006), tiene como objetivo indagar la incidencia y los valores en que se manifiesta una o más variables. La población de este estudio estuvo constituida por 50 docentes de básica primaria de la Institución Educativa No.9. del municipio Maicao, departamento La Guajira, Colombia, distribuidos de la siguiente manera:

Tabla 1

Distribución de la población

\begin{tabular}{llc}
\hline Sedes Educativa & No. Docentes \\
\hline 1. Manuel Rosado Iguarán & 18 \\
2. 20 de julio & 14 \\
3. Villa Inés & 12 \\
4. Gabriel García Márquez & 6 \\
TOTAL & 50 \\
\hline
\end{tabular}

Fuente: Elaboración propia

Es pertinente destacar que debido a que la población es accesible y finita, según Arias (2012), no fue necesaria la aplicación de técnicas de muestreo, se consideró un censo poblacional, encuetándose a la totalidad de los individuos, quienes facilitaron la información correspondiente para la presente investigación.

La técnica utilizada para la recolección de datos fue la encuesta, que según Arias (2012) es una técnica que pretende obtener información que suministra un grupo o muestra de sujetos acerca de si mismos, o en relación con un tema particular; el instrumento, un cuestionario de elaboración propia (2016), constó de cuarenta (40) ítems, con una escala tipo frecuencia de cinco (5) alternativas de respuesta, que contemplaba como opciones: Siempre (5), casi siempre(4), Algunas veces (3), casi nunca (2) Nunca (1). Dicho instrumento se sometió a la validez de cinco (5) expertos en el área de informática educativa y metodología de la investigación.

Por ende, el instrumento se sometió a un proceso de confiabilidad aplicándose la fórmula del Alfa Cronbach. El resultado obtenido fue una confiabilidad de 0.95 (altamente confiable), que confirma la muy alta confiabilidad del cuestionario diseñado para la población seleccionada para el presente estudio, en virtud de los criterios propuesto por Collado \& Hernández (2010), lo que demuestra la pertinencia y su aplicación.

El análisis y discusión de los resultados se efectuó en una fase; luego de su aplicación, los datos obtenidos se registraron en tablas que permitieron su análisis con el siguiente baremo: 
Tabla 2

Baremo de interpretación de las medias

\begin{tabular}{cccc}
\hline Grupo & Categorías & Valores & Categoría \\
\hline $\mathbf{5}$ & Siempre & $4,30-5$ & Muy alto \\
$\mathbf{4}$ & Casi siempre & $3,50-4,29$ & Alto \\
$\mathbf{3}$ & Algunas veces & $2,70-3,49$ & Moderado \\
$\mathbf{2}$ & Casi nunca & $1,90-2,69$ & Baja \\
$\mathbf{1}$ & Nunca & $1-1,89$ & Muy baja \\
\hline
\end{tabular}

Fuente: Elaboración propia

\section{Resultados}

Para cumplir con el objetivo de investigación planteado en la presente investigación, el cual es Identificar los tipos de comunidades virtuales de aprendizaje existente en la institución educativa No.9, sede Manuel Rosado Iguarán, 20 de Julio, Villa Inés y García Márquez del municipio de Maicao, La Guajira, Colombia, se procedió a la tabulación de todos los datos recolectados. Se analizan los resultados en su respectiva dimensión e indicadores. Para ello, se relacionan cinco alternativas de respuestas que permitirán relacionar los juicios emitidos por los encuestados.

Tabla 3

Resultados del indicador comunidades de interés

\begin{tabular}{|c|c|c|c|c|c|c|c|c|c|c|c|c|c|c|c|c|c|c|c|c|c|c|c|c|}
\hline \multirow[t]{3}{*}{ ALTERNATIVAS } & \multicolumn{6}{|c|}{ Comunidades de interés } & \multicolumn{6}{|c|}{$\begin{array}{l}\text { Comunidades de interés } \\
\text { inteligencia }\end{array}$} & \multicolumn{6}{|c|}{ Comunidades de aprendices } & \multicolumn{6}{|c|}{ Comunidades de práctica } \\
\hline & \multicolumn{2}{|c|}{ ITEMS 1} & \multicolumn{2}{|c|}{ ITEMS 2} & \multicolumn{2}{|c|}{ ITEMS 3} & \multicolumn{2}{|c|}{ ITEMS 4} & \multicolumn{2}{|c|}{ ITEMS 5} & \multicolumn{2}{|c|}{ ITEMS 6} & \multicolumn{2}{|c|}{ ITEMS 7} & \multicolumn{2}{|c|}{ ITEMS 8} & \multicolumn{2}{|c|}{ ITEMS 9} & \multicolumn{2}{|c|}{ ITEMS 10} & \multicolumn{2}{|c|}{ ITEMS 11} & \multicolumn{2}{|c|}{ ITEMS 12} \\
\hline & FA & FR & FA & FR & FA & FR & FA & FR & FA & FR & FA & FR & FA & FR & FA & FR & FA & FR & FA & FR & FA & FR & FA & FR \\
\hline Siempre & 20 & $40 \%$ & 14 & $28 \%$ & 18 & $36 \%$ & 32 & $64 \%$ & 8 & $16 \%$ & 0 & $0 \%$ & 0 & $0 \%$ & 35 & $70 \%$ & 35 & $70 \%$ & 31 & $62 \%$ & 12 & $24 \%$ & 19 & $38 \%$ \\
\hline Casi siempre & 15 & $30 \%$ & 17 & $34 \%$ & 11 & $22 \%$ & 14 & $28 \%$ & 25 & $50 \%$ & 21 & $42 \%$ & 14 & $28 \%$ & 0 & $0 \%$ & 0 & $0 \%$ & 4 & $8 \%$ & 21 & $16 \%$ & 18 & $36 \%$ \\
\hline Algunas veces & 0 & $0 \%$ & 8 & $16 \%$ & 17 & $34 \%$ & 4 & $8 \%$ & 17 & $34 \%$ & 14 & $28 \%$ & 17 & $34 \%$ & 8 & $16 \%$ & 0 & $0 \%$ & 4 & $8 \%$ & 28 & $16 \%$ & 0 & $0 \%$ \\
\hline Casi nunca & 7 & $14 \%$ & 11 & $22 \%$ & 4 & $8 \%$ & 0 & $0 \%$ & 0 & $0 \%$ & 15 & $30 \%$ & 19 & $38 \%$ & 7 & $14 \%$ & 7 & $14 \%$ & 11 & $22 \%$ & 0 & $0 \%$ & 13 & $26 \%$ \\
\hline Nunca & 8 & $16 \%$ & 0 & $0 \%$ & 0 & $0 \%$ & 0 & $0 \%$ & 0 & $0 \%$ & 0 & $0 \%$ & 0 & $0 \%$ & 0 & $0 \%$ & 8 & $16 \%$ & 0 & $0 \%$ & 9 & $18 \%$ & 0 & $0 \%$ \\
\hline \multirow[t]{2}{*}{ TOTAL } & 50 & $100 \%$ & 50 & $100 \%$ & 50 & $100 \%$ & 50 & $100 \%$ & 50 & $100 \%$ & 50 & $100 \%$ & 50 & $100 \%$ & 50 & $100 \%$ & 50 & $100 \%$ & 50 & $100 \%$ & 50 & $100 \%$ & 50 & $100 \%$ \\
\hline & 3,6 & & 3,7 & & 3,9 & & 4,6 & & 3,8 & & 3,1 & & 2,9 & & 4,3 & & 3,9 & & 4,1 & & 3,5 & & 3,9 & \\
\hline
\end{tabular}

Fuente: Elaboración propia

La tabla anterior permite apreciar la ponderación cuantitativa de los datos obtenidos mediante el instrumento, en lo que respecta a las comunidades de interés, se aprecia una cuantificación que no llega a la mitad una vez se analiza el apartado comunidades de interés, la situación es alarmante, dado que menos de la mitad afirma pertenecer a uno de estos grupos o en su defecto haber estado incorporado en el pasado, de igual forma se aprecia que la implementación de herramientas que fomenten la creación de comunidades de aprendizaje es baja, a esto se le añade que las comunidades existentes tienen finalidades superficiales y solo se acaricia el su potencial lo cual tiene su origen el desconocimiento o poca capacitación. En lo que respecta a las comunidades de interés inteligencia, el análisis de los datos indica que los docentes conforman grupos de trabajo encaminados a la mejorar la praxis educativa de la mano con las TIC. Sin embargo, los esfuerzos se debilitan por la poca disponibilidad a trabajar de forma interdisciplinar, lo cual es desalentador, dado que las comunidades virtuales en la institución son palpables pero estas prácticas individualistas repercuten de forma directa en el aseguramiento del aprendizaje. 
Una vez analizados los datos recolectados en el apartado de comunidades de aprendices se aprecia la predisposición de los docentes a trabajar de forma individual, lo cual va ligado a la falta de interacción entre colegas, la baja participación entre los profesores de diferentes áreas para crear e innovar lleva a un nivel bajo $\mathrm{n}$ de colaboración y creación de redes de aprendizaje virtuales. Por otra parte, los docentes encuestados manifiestan ser consciente de las falencias que tienen, para suplirlas realizan actividades encaminadas a mejorar su praxis pedagógica. Sin embargo, el intercambio de experiencias es bajo, lo que indica un ambiente de trabajo reacios en el que la interacción se limita a lo estrictamente laboral.

Tabla 4

Resultados de las medias aritméticas

\begin{tabular}{|c|c|c|c|c|}
\hline Indicadores & Interés & $\begin{array}{c}\text { Interés } \\
\text { Inteligente }\end{array}$ & Aprendices & De Práctica \\
\hline Media indicador & 3.7 & 3.8 & 2.9 & 4.1 \\
\hline Expresión cualitativa & Alto & Alto & Bajo & Alto \\
\hline Media de la dimensión & \multicolumn{3}{|c|}{ Moderado } \\
\hline Expresión cualitativa & \multicolumn{3}{|c|}{} \\
\hline
\end{tabular}

Fuente: Elaboración propia

Los datos que se expresan en la parte superior precisan aspectos cuantitativos y cualitativos, los cuales responden a ponderación obtenida luego de establecer los resultados correspondientes a la media altimétricas, así mismo, se observa de forma detallada que 3.7, una número considerable de los docentes de la IE No.9 hacen parte activa de comunidades virtuales de aprendizaje de tipo de interés común para los mismos; la comunidad de interés inteligente arrojó una media aritmética presenta un valor de 3,8; ello permite indicar que un grupo considerable de docentes de la IE No.9, hace parte de grupos para mejorar su praxis y adquirir nuevos conocimientos; fue entonces Alto según el baremo establecido. Con relación a la Comunidad de Aprendices, esta arrojó una media aritmética de 2.9, resultado que indica la baja tendencia de los docentes de la IE No.9 en hacer parte de las comunidades de aprendices.

Para concluir este apartado, en este ítem correspondiente a la media aritmética, en la cual se alcanzó un valor de 4,1; según lo establecido por el baremo, la gran parte de los docentes resalta de importancia de participar en espacios orientados a enriquecer su praxis a través de la mediación de las tecnologías de la información y la comunicación.

\subsection{Discusión}

Posterior al análisis de los resultados de la investigación y la confrontación de los datos con los apartados teóricos, se considera pertinente plantear que existe una comunidad de aprendices en la institución educativa, no obstante, se resalta que su articulación con el componente tecnológico es insuficiente, esto permite apreciar un escenario en el que los grupos de aprendizaje son vistos con una predisposición negativa. Dado que la apropiación y participación en comunidades virtuales de aprendizaje es baja, según los datos planteados en la tabla número tres del artículo. A priori, se torna complejo el desarrollo de actividades encaminadas a alcanzar objetivos, puesto que el trabajo no es de forma mancomunada.

Las comunidades virtuales de aprendizaje representan un puente para trascender de un escenario en el cual el trabajo en equipo tiene lugar bajo la tutela de la presencialidad y las categorías restrictivas como lo son, poco acceso a la información y velocidades de trabajo paquidérmicas, en la institución educativa intervenida mediante el estudio se consta que hay comunidades de aprendizaje cuyas acciones y actividades se desarrollan con ayuda de la tecnología, pero esto no indica que estas acciones sean constantes, dado que se acude a estos mecanismos 
solo cuando las circunstancias forzar a la comunidad académica, esto se aprecia al momento de tabular los datos de la encuesta. Pues se ve como última opción o no entra entre las primeras en el abanico de intervenciones.

Estos resultados son contrastados con las premisas de Meirinhos y Osório (2009), quienes señalan que la formación de comunidades de aprendizaje, orientadas para el desarrollo de procesos colaborativos, requiere de la creación de una cultura de participación en las actividades de sus miembros. Es decir, se refleja el esfuerzo de los actores implicados mediante la puesta en marcha de acciones como el compartir, participar y construir conjuntamente representaciones fiables y objetivas de nuevos conceptos. Por ende, es preponderante la creación de nuevos espacios encauzados a fomentar la participación pedagógica y el fortalecimiento de las competencias docente mediante la actualización constante.

\section{Conclusiones}

Partiendo de los planteamientos propuestos, siguiendo la línea metódica del análisis de los resultados posterior a la administración del instrumento al grupo muestral, se consignan las siguientes conclusiones, en este espacio es preponderante resaltar la identificación de los tipos de comunidades virtuales de aprendizaje subyacentes en la institución según las aseveraciones planteadas en la taxonomía propuesta por Meirinhos y Osório (2009), en la IE No.9, en orden de predilección por los docentes son: interés inteligente, interés, práctica y la de aprendices. Pese a la existencia de la comunidad de aprendices en la IE No.9, ésta se genera por la necesidad misma de la profesión docente y no es mediada por las tecnologías de la información y la comunicación.

Un sector de los docentes de la IE No.9, participan de forma activa en CVA para mejorar su práctica laboral. La resistencia al cambio es visible en algunos escenarios, dado que el nivel de implementación de las tecnologías de la información y la comunicación no es constante y por su reciente aparición en el contexto del trabajo grupal la confianza es poca. Del mismo modo, como parte de los resultados, se percibe el grado de apropiación de cuatro tipos de comunidades virtuales en la Institución Educativa No.9, sedes Manuel Rosado Iguarán, 20 de Julio, Villa Inés y Gabriel García Márquez del municipio de Maicao, La Guajira, Colombia, las cuales son detalladas en la parte superior del escrito. Dicha categorización permitió evidenciar una tendencia negativa hacia las comunidades de aprendices, aun cuando se ubica en la expresión es baja, mientras la de práctica, de interés e interés inteligente se observó con una expresión alta por los docentes encuestados.

Para concluir, subyace la necesidad de un firme y regular proceso de capacitación al personal docente en el manejo e implementación de las Tecnologías de la Información y la Comunicación (TIC) para motivar e incentivar el trabajo colaborativo y de esta forma dar lugar a la conformación de CVA, redes de aprendizaje y conocimiento, lo cual de forma causal exhorta a la participación e innovación en base a las necesidades del entorno en el cual se enmarca el docente.

\section{Declaración de conflictos}

Para la redacción del presente artículo de investigación se manifiesta que los autores no tienen intereses ajenos a publicar y dejan constancia no han incurrido en actuaciones indebidas para lograr tal fin.

\section{Referencias bibliográficas}

Arias, F. (2012). El Proyecto de investigación, introducción a la metodología científica. 6ta edición. Caracas: Episteme

Cabero Almenara, Julio. (2010). El aprendizaje autorregulado como marco teórico para la aplicación educativa de las comunidades virtuales y los entornos personales de aprendizaje. Revista: Teoría de la Educación. 
Educación y cultura en la sociedad de la información. Universidad de Salamanca. TESI, 14(2), 2013, pp. 133-156.

CABERO,"J."(2006)."Comunidades"virtuales"para"el"aprendizaje."Su"utilización"en"la"enseñanza. Edutec,\& 20. Extraído" el" 20" de" enero" " de" 2008" desde "'" http://www.uib.es/depart/gte/gte/edutecle/revelec20/cabero20.htm

Canales Reyes, R., \& Marquès Graells, P. (2007). Factores de buenas prácticas de enseñanza con apoyo de las TIC. El análisis de su presencia en tres escuelas. Educar, (39), 115-133.

Cejas-León, R., \& Navío-Gámez, A. (2020). Sobre la formación tecnopedagógica del profesorado. La visión de los expertos y formadores. Revista iberoamericana de educación superior, 11(31), 150-164.

Coll, César. (2008). Psicología de la educación virtual: aprender y enseñar con las tecnologías de la información y la comunicación. Ediciones Moratas SL. 12/29004. Madrid España.

Hernández Sampieri, R., Fernández Collado, C., \& Baptista Lucio, P. (2014). Metodología de la investigación (6a. ed. --.). México D.F.: McGraw-Hill..

Alejandro De Valenzuela del Aguila, A. (2013). Herramientas TIC que potencian el trabajo en equipo.

Díez-Palomar, J., \& García, R. F. (2010). Comunidades de Aprendizaje: un proyecto de transformación social y educativa. Revista interuniversitaria de formación del profesorado, 24(1), 19-30.

Dillenbourg, P., Poirier, C., \& Carles, L. (2003). Communautés virtuelles d'apprentissage: e-jargon ou nouveau paradigme. A. Taurisson et A. Sentini. Pédagogies. Net. Montréal, Presses, 11-47.

Gairín Sallán, Joaquín. (2006). Las comunidades virtuales de aprendizaje. Educar 37, 2006 41-64..

Muñoz-Repiso, A. G.-V. (2016). Las competencias digitales en el ámbito educativo.

Gros, B. (2008). El aprendizaje colaborativo a través de la red: límites y posibilidades. Sólo para uso Docente Distribución Gratuita, 112.

Hernández Sampieri, Roberto; Fernández Collado, Carlos y Lucio Batispta, Pilar. (2006). Metodología de la investigación. 4ạ Edición. Editorial MacGraw colina. México.

Levano-Francia, L., Sanchez Diaz, S., Guillén-Aparicio, P., Tello-Cabello, S., Herrera-Paico, N., \& Collantes-Inga, Z. (2019). Competencias digitales y educación. Propósitos y representaciones, 7(2), 569-588.

Meirinhos, Manuel \& Osório, António. (2009). Las comunidades virtuales de aprendizaje: el papel central de la colaboración. Pixel-Bit. Revista de Medios y Educación. № 35 Julio 2009 pp.45 - 60.

Martín, A. H., \& Rodríguez, A. I. (2017). La importancia de las competencias digitales e informacionales para el desarrollo de una escuela intercultural. Interacçoes, 13(43).

MINISTERIO DE EDUCACIÓN NACIONAL (2012). Política y sistema colombiano de formación y desarrollo profesional de educadores, MEN, Colombia.

Muñoz, I. D. (2014). El trabajo en equipo y las TICs en la enseñanza de traducción especializada. TIC, trabajo colaborativo e interacción en Terminología y Traducción, 71.

Organización de las Naciones Unidas para la Educación, la Ciencia y la Cultura -UNESCO-. (2013). Análisis del Clima Escolar. ¿Poderoso factor que explica el aprendizaje en América Latina y el Caribe. Laboratorio Latinoamericano de Evaluación de la Calidad de la Educación 
Tirado Morueta, Ramón \& Martínez Garrido, Juan Manuel. (2010). Creando comunidades virtuales de aprendizaje: análisis del progreso de las interacciones. Universidad de Huelva. Facultad de Ciencias de la Educación. Departamento de Educación. Huelva, España.

Tovar-Gálvez, J. C. (2017). Pedagogía ambiental y didáctica ambiental: tendencias en la educación superior. Revista Brasileira de Educação, 22(69), 519-538.

Velázquez, A. K. O., \& Cupil, R. G. (2020). LA RESIGNIFICACIÓN PROFESIONAL A TRAVÉS DE LA FORMACIÓN EN COMPETENCIAS DIGITALES. Presencia Universitaria, (12), 74-85.

Z Vega, C. Z., Fallas, J. G., Serrano, E. A., Rojas, L. M. H., Delgado, M. D. L. Á. C., Esquivel, C. C., ... \& Quirós, S. A. (2011). Construcción de una comunidad virtual de aprendizaje ambiental (CVAA): espacio interactivo para ambientalizar el quehacer universitario estatal de Costa Rica/Building a virtual community for environmental learning (VCEL): an interactive space to... Actualidades Investigativas en Educación, $11 .$.

\section{Anexos}

\section{Anexo A. Instrumento para la recolección de datos}

\section{INSTRUMENTO PARA LA RECOLECCIÓN DE LA INFORMACIÓN}

\begin{tabular}{|c|c|c|c|c|c|}
\hline \multirow{3}{*}{$\begin{array}{l}\text { COMUNIDAD VIRTUAL DE APRENDIZAJE COMO ESTRATEGIA PARA LAS PRÁCTICAS } \\
\text { DOCENTES }\end{array}$} & \multicolumn{5}{|c|}{ ALTERNATIVA } \\
\hline & $\frac{\text { o }}{\frac{\varrho}{\varrho}}$ & $\overline{\bar{U}}$ & 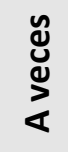 & $\overline{\bar{u}}$ & 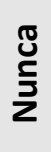 \\
\hline & 5 & 4 & 3 & 2 & 1 \\
\hline $\begin{array}{l}\text { VARIABLE: COMUNIDAD VIRTUAL DE APRENDIZAJE COMO ESTRATEGIA PARA LAS PRÁCTICAS } \\
\text { DOCENTES }\end{array}$ & $\mathbf{S}$ & CS & AV & CN & $\mathbf{N}$ \\
\hline \multicolumn{6}{|l|}{ DIMENSIÓN:TIPOS DE COMUNIDADES VIRTUALES DE APRENDIZAJE } \\
\hline \multicolumn{6}{|l|}{$\begin{array}{l}\text { INDICADOR:COMUNIDAD DE INTERÉS } \\
\text { Usted como docente... }\end{array}$} \\
\hline 1. Es miembro activo de una comunidad virtual de aprendizaje. & & & & & \\
\hline $\begin{array}{l}\text { 2. Utiliza una comunidad virtual de aprendizaje para intercambiar información entre los } \\
\text { miembros de dicha comunidad. }\end{array}$ & & & & & \\
\hline $\begin{array}{l}\text { 3. Considera que los miembros de su comunidad virtual de aprendizaje comparten temas de } \\
\text { interés común. }\end{array}$ & & & & & \\
\hline \multicolumn{6}{|l|}{ INDICADOR:COMUNIDAD DE INTERÉS INTELIGENTE } \\
\hline $\begin{array}{l}\text { 4. En la institución educativa existen grupos con objetivos comunes según el área de } \\
\text { conocimiento. }\end{array}$ & & & & & \\
\hline 5. Conforma grupos de trabajo para generar conocimiento científico. & & & & & \\
\hline 6. Realiza intercambios de experiencias con sus pares en función del bien común. & & & & & \\
\hline \multicolumn{6}{|l|}{ INDICADOR: COMUNIDAD DE APRENDICES } \\
\hline 7. Participa en comunidades virtuales de aprendizaje para construir conocimiento. & & & & & \\
\hline 8. Interactúa con sus pares para ampliar el intercambio de conocimiento. & & & & & \\
\hline 9. $\quad$ Práctica el aprendizaje colaborativo entre sus pares. & & & & & \\
\hline \multicolumn{6}{|l|}{ INDICADOR: COMUNIDAD DE PRÁCTICA } \\
\hline 10. Participa en comunidades de práctica en función de mejorar su praxis como docente. & & & & & \\
\hline $\begin{array}{l}\text { 11. Intercambia saberes en las comunidades de práctica con profesionales de otras áreas de } \\
\text { conocimiento. }\end{array}$ & & & & & \\
\hline
\end{tabular}


COMUNIDAD VIRTUAL DE APRENDIZAJE COMO ESTRATEGIA PARA LAS PRÁCTICAS DOCENTES

VARIABLE: COMUNIDAD VIRTUAL DE APRENDIZAJE COMO ESTRATEGIA PARA LAS PRÁCTICAS DOCENTES

12. Participa de forma frecuente en discusiones en una comunidad virtual hasta encontrar la solución colectiva del problema.

\section{DIMENSIÓN: CARACTERÍSTICAS DE LAS COMUNIDADES VIRUTALES DE APRENDIZAJE}

INDICADOR: COMPROMISO MUTUO

13. Negocia con sus pares la solución de algún conflicto en la comunidad virtual de aprendizaje en la que participa.

14. Considera que en su comunidad virtual se relacionan entre sí.

15. Considera que participar en una comunidad virtual de aprendizaje le permite desarrollar su praxis como docente.

\section{INDICADOR: EMPRENDIMIENTO CONJUNTO}

16. Practica la negociación colectiva en la comunidad virtual de aprendizaje que pertenece.

17. Se compromete a realizar las actividades antes de dar inicio a la misma.

18. Crea entre los participantes de la comunidad virtual relaciones de responsabilidad mutua. INDICADOR: REPERTORIO COMPARTIDO

19. Evita conflictos dentro de la comunidad virtual de aprendizaje en su praxis como docente.

20. Socializa en la comunidad virtual de aprendizaje con los nuevos miembros temas de interés común.

21. Desarrolla recursos que comparte con sus pares en la comunidad virtual de aprendizaje.

\section{DIMENSIÓN No.3: DIMENSIÓN INTERPERSONAL}

\section{INDICADOR: CLIMA ESCOLAR}

22. Realiza su práctica docente en el marco de mantener un nivel de convivencia con sus estudiantes.

23. Considera que el clima institucional permite la construcción de vivencias socio-afectivas entre sus actores.

24. Considera que existe calidad en las relaciones interpersonales entre los actores del proceso educativo.

\section{INDICADOR: ESPACIOS DE PARTICIPACIÓN INTERNA}

25. Considera que dentro de la institución existan espacios para promover la confianza entre los actores del proceso educativo.

26. Considera que dentro de la institución educativa se manejan espacios agradables de trabajo.

27. Utiliza la participación democrática en la toma de decisiones de las actividades que desea realizar.

\section{INDICADOR: CONVIVENCIA ESCOLAR}

28. Contribuye a la construcción colectiva de la convivencia escolar.

29. Participa en la creación de actividades que generan la convivencia escolar.

30. Realiza una distribución equitativa del poder para generar convivencia escolar.

DIMENSIÓN: DIMENSIÓN DIDÁCTICA

INDICADOR: MÉTODOS DE ENSEÑANZA

31. Facilita el aprendizaje de los estudiantes en la construcción de su propio conocimiento.

32. Los momentos de la clase permiten en los estudiantes la adquisición de conocimiento a través de la exploración de situaciones.

33. Considera que los métodos utilizados en su praxis fomenta el aprendizaje.

INDICADOR: ORGANIZACIÓN DEL AULA DE CLASES 


\begin{tabular}{|c|c|c|c|c|c|}
\hline \multirow{3}{*}{$\begin{array}{l}\text { COMUNIDAD VIRTUAL DE APRENDIZAJE COMO ESTRATEGIA PARA LAS PRÁCTICAS } \\
\text { DOCENTES }\end{array}$} & \multicolumn{5}{|c|}{ ALTERNATIVA } \\
\hline & 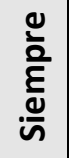 & $\overline{\tilde{U}}$ & 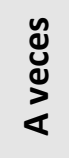 & $\overline{\mathfrak{u}}$ & $\begin{array}{l}\text { J } \\
\text { ¿ } \\
z\end{array}$ \\
\hline & 5 & 4 & 3 & 2 & 1 \\
\hline $\begin{array}{l}\text { VARIABLE: COMUNIDAD VIRTUAL DE APRENDIZAJE COMO ESTRATEGIA PARA LAS PRÁCTICAS } \\
\text { DOCENTES }\end{array}$ & $\mathbf{S}$ & CS & AV & CN & $\mathbf{N}$ \\
\hline 34. Organiza el trabajo en el aula por grupos para promover el aprendizaje colaborativo. & & & & & \\
\hline $\begin{array}{l}\text { 35. Se distribuye el aula de clases en forma adecuada para motivar la permanencia de los } \\
\text { estudiantes. }\end{array}$ & & & & & \\
\hline $\begin{array}{l}\text { 36. Organiza trabajos individuales que permitan evaluar el desempeño académico de los } \\
\text { estudiantes. }\end{array}$ & & & & & \\
\hline INDICADOR: ESTILOS DE APRENDIZAJE & & & & & \\
\hline $\begin{array}{l}\text { 37. Toma en cuenta las características personales de cada estudiante en el desarrollo de su } \\
\text { praxis. }\end{array}$ & & & & & \\
\hline 38. Considera las distintas formas de aprender de los estudiantes. & & & & & \\
\hline 39. Toma en cuenta las diferentes formas de percibir el conocimiento de los estudiantes. & & & & & \\
\hline
\end{tabular}

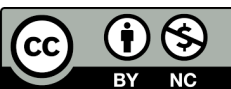

Esta obra está bajo una Licencia Creative Commons Atribución-NoComercial 4.0 Internacional 